\title{
Bridging the gap Why we need to enhance current simulation models
}

\author{
Kneidl Angelika \\ accu:rate $\mathrm{GmbH}$ \\ Rosental 5, Munich, Germany \\ ak@accu-rate.de
}

\begin{abstract}
Many models that simulate evacuations are state of the art and provide realistic insight to their users. However, simulating everyday situations, such as visitor flow through a museum or passenger flow through an airport, presents marked challenges; existing models reach their limit here. This contribution will introduce and highlight the gap between existing egress models and the difficulties found simulating, for instance, passenger flow or capacity analysis.
\end{abstract}

Keywords: pedestrian simulation model enhancements, agent-based behaviour, persona approach, simulation of everyday situations

\section{Introduction}

Over the last few decades, a variety of different pedestrian simulation models have been developed. These models range from the physics-inspired Social Force Models ([3]), to Cellular Automaton [5] that have their origin in traffic simulation, and agent-based models that base their ideas on computer game engines ([1], [6]). In between, many hybrid models combine aspects of each approach. Each of these models have their strengths and weaknesses. [11] present a thorough overview of existing models.

Since the 90s, the number of use-cases for such models has grown beyond evacuation analyses: the more advanced the models, the more the demand from practitioners.

This work was inspired from the $S^{2} U C R E$ research project (www.s2ucre.de): Here, we try to predict the short-time future from video footage. Since we only get aggregated data and have to extract individuals, modelling of behaviours is essential to a most realistic result. This context refers to only as one example current simulation models need to be improved to model crowds more realistically.

In this paper, impetus to develop existing simulation models towards market needs shall be provided.

\section{State-of-the-Art Models: What are they capable of and what we need}

To model pedestrian behaviour most accurately, models should consider different layers of intent [6]:

The strategy layer describes a pedestrian's motivation for movement. For instance: a commute to work, hurrying to a meeting, or a stroll through a museum. This layer should not be modelled explicitly. Rather, it helps derive simulation parameters, such as speed or torso size.

The navigational layer describes how pedestrians move from their current position to a location that is not visible to them.

The locomotion layer models the stepping (or in some models sliding) of a pedestrian. It determines the best (though not necessarily optimal) next position based on, typically, a destination.

On top of these layers, behavioural characteristics must be taken into account. In microscopic pedestrian simulations, individuals are often described as just that: individual. However, it is well understood that people socialize with one another, resulting in behaviour that might not otherwise appear. Common examples are grouping behaviours and queues (e.g. [7], [8], [9]). Further, we learn from psychologists and 
sociologists that there are many more stimuli that influence our behaviour. [12] speak of Human Factors. There are four main factors they refer to:

- The environment / infrastructure we are moving in

- $\quad$ Any impacts (e.g. weather, fire, surroundings)

- Occasion (what is the reason for gathering or moving? This is the analogous to the strategy layer and thus for the choice of parameters)

- Humans (affiliation: what role am I on the occasion, e.g. family, security, friend...)

To include these factors, common simulation models must be extended. Furthermore, we have found that patterns describing individual mannerisms (e.g. politeness, impatience, anxiousness) and urges (e.g. needing the toilet, hunger, thirst) should be considered in modern simulation tools as well.

Naturally, these behaviours must not play a role in evacuation simulations, but must feature in business-as-usual simulations.

There exists already work which tries to cope with psychological factors and suggest models, like the PECS Model [13], PMServ[14] or a concurrent hierarchical state machine [15]. The PECS model is capable of modelling physical, emotional, cognitive and social factors and originates in Operations Research. The MACES (Multi-Agent Communication for Evacuation Simulation) in combination with PMServ (psychological representation with memory data in combination with a short-term memory system) is an architecture to integrate a psychological model into crowd simulations [14]. The authors of [15] propose a concurrent hierarchical finite state machine for modelling pedestrian behavioural tendencies.

These existing models try to model the complex interrelations between cognition, psychology and movement. They are only theoretical and academic approaches not set into practice so far and consist of very complex models. For practitioners, the objective is a simple model to integrate complex human behaviour. Thus, in the following chapter the most important aspects from a practitioners' point of view are addressed in order to narrow down the complexity.

\section{Needs from practitioners' point of view}

The requirements and wishes of the commercial sector are varied. Some of them are beyond the abilities of simulation models, but we have identified numerous gaps that can be closed by modern tools.

\subsection{How can we apply realistic avoidance behaviour for agents when it comes to counter- flows?}

Common simulation models tackle this situation with repelling and attracting forces. That is, agents walking against me are more repellent than agents who walk with me. Manifesting these phenomena as parameters and forces, simulation models can reproduce the observed lane formation that occurs when agents are in counter-flow. Still, with high densities, agents get stuck. In Fig. 1, different results for different repulsion setups are shown. 

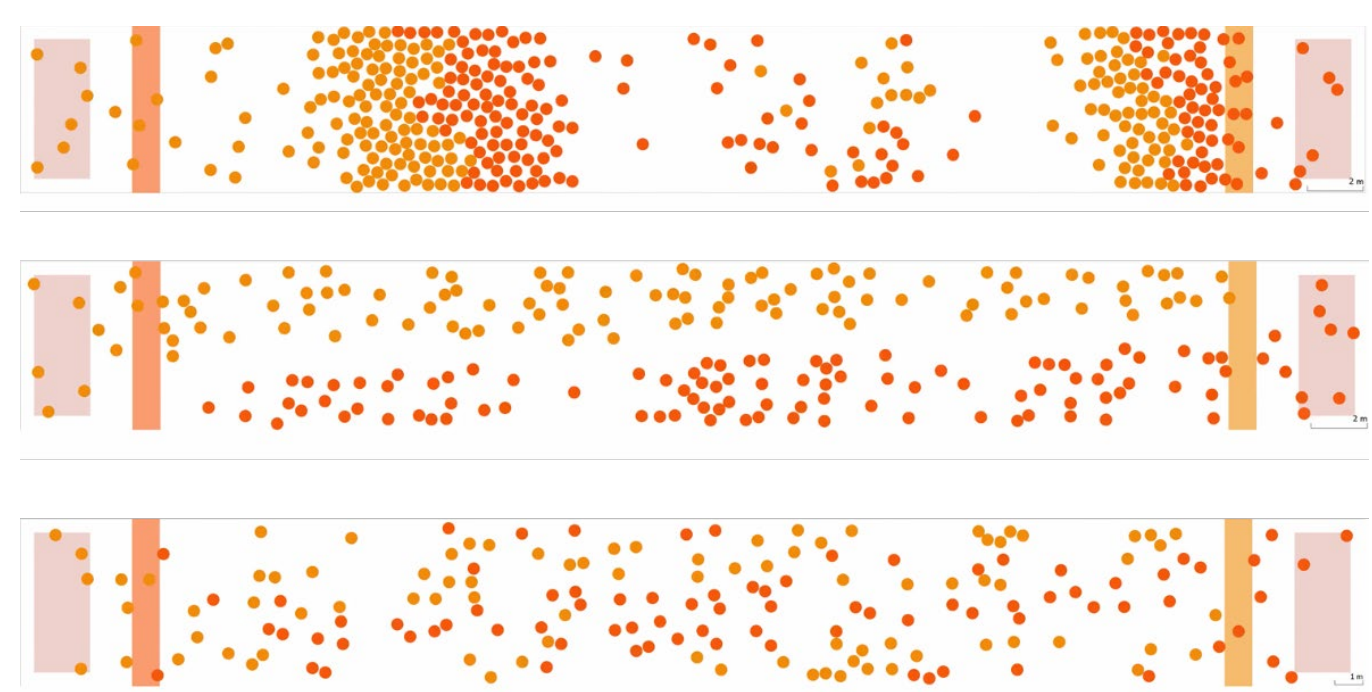

Fig. 1 Different results of counter-flow and lane formation: The upper figure shows results of very low counterflow repulsion resulting in blockage, the middle picture with a very high value where clear lanes occur, the lower picture shows a moderate repulsion.

The different outcomes in Fig. 1 show the challenge quite well: the same situation with the exact same number of agents lead to different results and lane formations when changing the repulsiveness of counterflow agents. Does it make sense to model counter-flows by adding repellent values or might it be better to model the behaviour of agents instead? The observed counter-flow patterns form due to behaviour of individuals: Some people are more polite and offer room for one another and even step back to let others pass whereas some people really try to squeeze through a crowd. By applying individual behaviours to agents, such counter-flow formations may evolve more or less intense depending on the agent types one would model and thus patterns would evolve from individuals' behaviours instead of forces that are applied on all pedestrians the same way.

\subsection{How can we teach agents Generalized Knowledge [2]?}

When modelling and simulating complex buildings, agents must "know" patterns of behaviour, which are learnt over time. I.e. if you are in a hospital, you do not need to search for an exit in a patient room, as you know there won't be an exit there; corridors will most likely guide you to an exit. An example of applying this knowledge is given in Fig. 2. Here, the agent chooses the shortest path (left) whereas in the right picture, he uses the knowledge that rooms won't lead to exits.

This implicit knowledge should be integrated into simulation models in order to present more realistic results - not only in emergency situations. First approaches have already been realized by [2]. Nevertheless, we may be able to extract semantic information from building information model, and use this extra layer of information in the simulation tools. Individual agents can then use semantic data uniquely to make decisions. But to what extent could these data be used? How much knowledge has everyone and how much does he make use of it? These are open questions to be answered when integrating knowledge into simulation tools. 


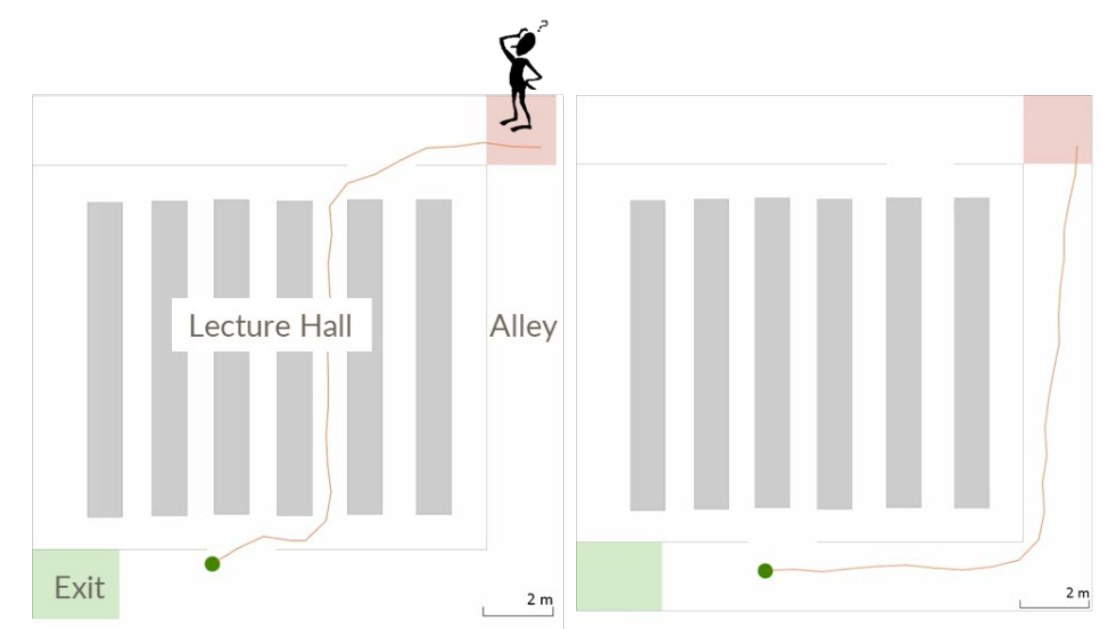

Fig. 2 Example of Generalized Knowledge: left, the agent has no generalized knowledge and walks through a lecture hall, since this is the shortest and fastest path; right, the agent walks along the corridor.

\subsection{What measures can we take to simulate correct behaviour in front of bottlenecks?}

Whenever many people share limited space, at some point bottlenecks may occur. Behaviour in front of bottlenecks depends on human factors, such as: Am I in a hurry? What situation am I in? Can I see why a congestion has occurred? As a consequence, queuing and waiting behaviour depends on the situation and the people involved in that situation. We have to ascertain which factors most influence behaviour in front of bottlenecks. Is it an intrinsic personal attitude that lets someone be more polite versus being more pushy, or are there extrinsic factors, such as the situation and possible danger that you face? Or is it a combination of both? How can these factors be translated into parameters we can use as input to our simulation models?

Another aspect is the decision on how long a pedestrian would queue for a certain point of interest, like a toilet or a ticket office. When would a person rethink and re-decide not using that facility but maybe search for another one? Same holds true for attractions one has to queue for.

A common approach to these issues is to tag certain environments as attractive or not. As such, people avoid or prefer certain areas depending on the attractiveness. However, this does not allow individual preferences or states. We therefore need to focus more on modelling individual human factors than labelling areas with an attractiveness.

Many models do not take the above behaviours into account but instead use statistics. We believe the integration of a persona model [10], defining a scale of urges and preferences would lead to greater realism and widen the purview of pedestrian simulation tools.

A key factor in these approaches however, is to generate an appropriate validation method, such that the quality of the model is assured.

\section{The road onwards: Integrating Machine Learning?}

This paper gives an overview on the gap between current state-of-the-art models and commercial consumer needs. As shown, pedestrian simulation applications have varied requirements. Tackling these requirements represents challenges for research: integration of results from psychological studies in order to be able to focus on agents and their individual behaviours. Until now, computers need a very specific set of rules, whereas psychologists and sociologists tend to give more vague answers and tendencies. New methods for tackling this discrepancy could be found in machine learning. When using such techniques, video input data can serve as training data and certain questions like "Where will a crowd move towards?" or "Do people use restrooms in the first floor more likely than in the second floor?" addressed. 
Integrating such new methods into a hybrid model, where we use a Persona model for modelling certain aspects like the motivation of pedestrians plus some basic characteristics like patience, preferences, mobility, grouping in combination of "learned" patterns from crowds may lead to a new generation of simulation models that are able to bridge the gap.

\section{Acknowledgements}

This work was funded by the German Federal Ministry of Education and Research through the project S2UCRE (grant number 13N14464). 


\section{References}

[1] Funge, John and Tu, Xiaoyuan and Terzopoulos, Demetri, "Cognitive Modeling: Knowledge, Reasoning and Planning for Intelligent Characters", Proceedings of the 26th annual conference on Computer graphics and interactive techniques, p-29-38, 1999

[2] Andresen, E., M. Charaibi, A. Seyfried, "A Representation of Partial Spatial Knowledge: A Cognitive Map Approach for Evacuation Simulations”, Transportmetrica A: Transport Sciene, pp.1-18, 2018

[3] Moussaïd, M. \& Helbing, D.,How simple rules determine pedestrian behavior and crowd disasters,Proceedings of the National Academy of Sciences, 2011, 108, 6884-6888

[4] Helbing, D. \& Molnár, P.,Social Force Model for Pedestrian Dynamics Physical Review E, 1995, 51, 4282-4286

[5] Blue, V.\& Adler, J.,Cellular Automata Microsimulation of Bidirectional Pedestrian Flows, Transportation Research Record: Journal of the Transportation Research Board, 1999, 1678, 135-141

[6] Reynolds, C.,Steering Behaviors for Autonomous Characters, Game Developers Conference, 1999

[7] Köster, G.; Treml, F.; Seitz, M. \& Klein, W.,Validation of crowd models including social groups,Proceedings of the 6th International Conference on Pedestrian and Evacuation Dynamics, 2012

[8] Moussaïd, M.; Perozo, N.; Garnier, S.; Helbing, D. \& Theraulaz, G., The Walking Behaviour of Pedestrian Social Groups and Its Impact on Crowd Dynamics, PLoS ONE, 2010, 5, e10047

[9] Köster, G. \& Zönnchen, B.,Queuing at Bottlenecks Using a Dynamic Floor Field for Navigation, Transportation Research Procedia , 2014, 2, 344 - 352

[10] C. Schaefer, R. Zinke, L. Kuenzer, G. Hofinger, and R. Koch, “Applying Persona method for describing users of escape routes?" in The Conference on Pedestrian and Evacuation Dynamics 2014 (PED2014), 2014, pp. 636-641.

[11] D. C. Duives, W. Daamen, and S. P. Hoogendoorn, "State-of-the-art crowd motion simulation models," Transportation Research Part C: Emerging Technologies, vol. 37, pp. 193-209, 2013

[12] G. Hofinger, R. Zinke, and L. Künzer, "Human Factors in Evacuation Simulation, Planning, and Guidance," Transportation Research Procedia, vol. 2, pp. 603-611, 2014

[13] B. Schmidt: Modelling of Human Behaviour: "The PECS Reference Model." AAAI Technical Report FS-01-02, 2001

[14] N. Pelechano, K. O’Brien K. B. Silverman, N. Badler, “Crowd Simulation Incorporating Agent Psychological Models, Roles and Communication“, First International Workshop on Crowd Simulation, 2005

[15] P. Kielar, O. Handel, D. Biedermann, A. Borrmann: "Concurrent hierarchical finite state machines for modeling pedestrian behavioral tendencies." Transportation Research Procedia 2 (2014) 576 - 584, 2014 